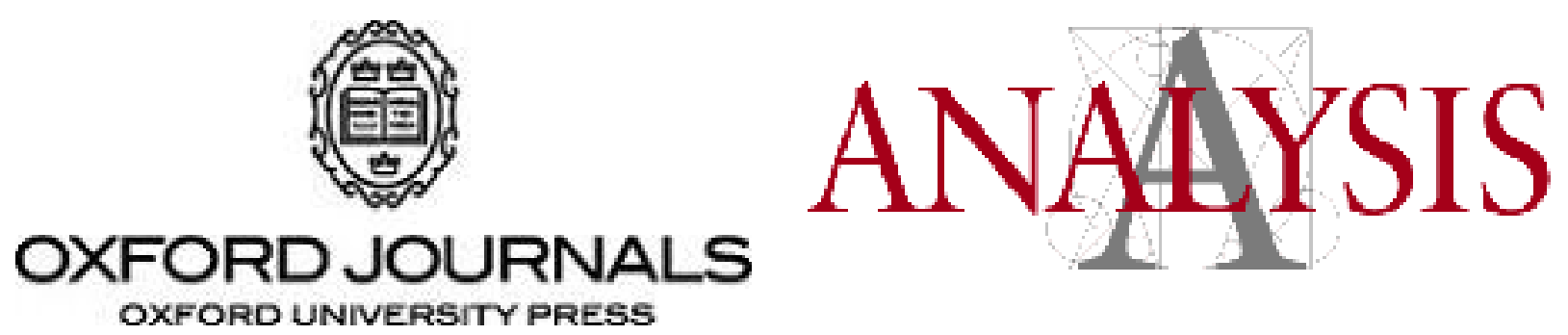

Comparatives and Degrees

Author(s): Adam Morton

Source: Analysis, Vol. 44, No. 1 (Jan., 1984), pp. 16-20

Published by: Oxford University Press on behalf of The Analysis Committee

Stable URL: http://www.jstor.org/stable/3327445

Accessed: $11 / 03 / 2014$ 16:30

Your use of the JSTOR archive indicates your acceptance of the Terms \& Conditions of Use, available at

http://www.jstor.org/page/info/about/policies/terms.jsp

JSTOR is a not-for-profit service that helps scholars, researchers, and students discover, use, and build upon a wide range of content in a trusted digital archive. We use information technology and tools to increase productivity and facilitate new forms of scholarship. For more information about JSTOR, please contact support@jstor.org.

Oxford University Press and The Analysis Committee are collaborating with JSTOR to digitize, preserve and extend access to Analysis. 
is tall' being an obvious ellipsis for ' _ _ is taller than the average', or the like. But ' _ is taller than ...' can only be interpreted as a predicable if it is taken as elliptical for ' _. is taller than ... is', and it is difficult to see how this predicable could occur in '_- is taller than ... was'. If we took ' - is taller than . . . was' as the result of subjecting the second 'is' in ' - is taller than .... is' to the past tense operator 'it was the case that _-' (as equivalent to '_- is taller than it was the case that ... is'), we should have to distinguish this from 'It was the case that _ is taller than . . . is', just as 'Bill was shorter than it has come to pass that Tom is' has to be distinguished from 'It has come to pass that Bill is shorter than Tom'. But how can there be two different ways of attaching a tense operator to a predicable? Traditional grammar counts 'than' as a conjunction, and if we were able as logicians to place it in the same syntactical category as, say, '_- or ...', which also counts as a conjunction, we could analyse "- is taller than it was the case that ... is' in the same way as '__ is white or it was the case that... is square'. But ' $\ldots$ is square' is already a complete predicable, whereas ' - is' is merely the copula, a word which normally has no function other than that of converting a noun or an adjective into a verb phrase. What is the second 'is' doing in '_- is taller than ... is'? I do not know the answer to this question, or indeed to the whole question about the correct analysis of propositions involving comparatives, and would like to use the pages of ANALYSIs to appeal for suggestions. One such suggestion appears on the following pages.

University of Bristol, Queens Road, Bristol BS8 1RJ

(c) C. J. F. WiLliams 1984

\title{
COMPARATIVES AND DEGREES
}

\author{
By ADAM Morton
}

\section{DEGREES}

THERE are many sentences in which the comparative form of the adjective cannot be taken as expressing a simple relation between the objects compared. For example
(a) Roger is fatter than we thought possible.
(b) Tony is fatter than he was yesterday.
(c) Tom has grown to be fatter than Bill.
(d) John ran faster than the Olympic record.
(e) Dennis drank more than the legal limit. 
(b) and (c) present Christopher Williams' main worry, but the problem is rather more general. It is: how can we explain the logical form of comparatives in a way which makes (a) to (e) and the familiar 'Tom is fatter than Bill' all instances of the same construction?

Here is a possible solution. Sentences like (d) and (e) above are revealing because 'the Olympic record' names a speed (a distance plus a time) and 'the legal limit' names a quantity of alcohol. In some cases, then, we can construe a comparative as relating a subject to a degree to which it does or does not instantiate a predicate. Thus (d) and (e) may be construed as

$\left(\mathrm{d}^{\prime}\right) \exists s$ (John ran at speed $s$ and $s$ exceeded the Olympic record)

(e') $\exists p \exists q$ (Dennis drank $p$ pints and it is illegal to drink more than $q$ pints and $p$ is greater than $q$ )

This strategy will work in general. (a) and (b) can be taken as

(a') $\exists g \exists$ (Roger's girth was $g$ and we did not think it possible that Roger's girth was greater than $h$ and $g$ exceeds $h$ )

(b') $\exists g \exists h$ (Tony's girth is now $g$ and Tony's girth was $h$ yesterday and $g$ is greater than $h$ )

$\left(b^{\prime}\right)$ shows how cross-temporal comparisons are possible.

Comparatives do appeal to two-place relations as thus construed, but not two-place relations between the things to which the corresponding non-comparative adjectives apply. The relation is between degrees. It is interesting to note that when the underlying non-comparative is two-place, the relation between degrees in the comparative is still only two-place, although there is a definite four-place appearance to the surface structure. Thus

(f) Adam loves Beth more than Carol loves David. is represented as

$\left(\mathrm{f}^{\prime}\right) \quad \exists d \exists e$ (Adam loves Beth to degree $d$ and Carol loves David to degree $e$ and $d$ is greater than $e$ ).

\section{PARTIAL ORDERINGS}

This last example should provoke a very natural worry. What are degrees and when is one greater than another? Love certainly does not come in numerical degrees, and it is for that matter far from obvious that fatness does either.

The relation between greater and lesser degrees of a predicate is not entirely unconstrained, though. It is evident that, for example:

$\mathrm{x}$ is $\mathbf{F}$-er than $\mathrm{y} \supset \mathrm{y}$ is not $\mathrm{F}$-er than $\mathrm{x}$

$x$ is not $F$-er than $x$

( $\mathrm{x}$ is F-er than $\mathrm{y}$ and $\mathrm{y}$ is F-er than $z$ ) $\supset \mathrm{x}$ is F-er than $\mathrm{z}$. 
These do not entail that degrees correspond to numbers. Their force can be quite easily summed up, though, by the assumption that degrees form partial orderings. That is, we may assume that the degrees of any predicate are ordered by a relation that is reflexive, antisymmetric (i.e. Rxy and Ryx imply $x=y$ ) and transitive. An abstract partial ordering can be taken as the pattern common to a set of partially ordered domains which can be out into an orderpreserving one-to-one correspondence. ${ }^{1}$ The importance of an abstract partial ordering for our purposes is that it is not affected by the accidental nonexistence of elements having a particular predicate to a particular degree. There is a great variety of (abstract) partial orderings. In most of them there are incomparable elements, elements none of which bears $R$ to any other. The representation of degrees by elements of partial orderings is thus compatible with the obvious possibility that $x$ is $F$ to degree $d$ and $y$ is $F$ to degree $e$, and neither $d$ nor $e$ exceeds nor equals the other.

So, to put these pieces together, let me introduce just one more piece of apparatus, in the form of a function $\mathcal{L}$ which correlates with each predicate $F$ its characteristic partial ordering $\mathcal{L}(\mathrm{F})$. Then the writing-out of ' $a$ is $\mathbf{F}$-er than $b$ was' is

$\exists d \exists e \exists t$ ( $a$ is $\mathrm{F}$ to degree $d$ now and $b$ is $\mathrm{F}$ to degree $e$ at time $t$ and $d$ exceeds $e$ in $\mathcal{L}(\mathrm{F})$ and $t$ is earlier than now)

and (e) 'John ran faster than the Olympic record' is

$\exists d$ (John ran fast to degree $d$ and the Olympic record is $e$ and $d$ exceeds $e$ in $\mathcal{L}$ (running fast)).

It should be clear how all of (a) to (e) and Williams' examples are dealt with in these terms.

\section{WHAT PARTIAL ORDERINGS?}

The virtue of this approach is that it provides a uniform reading of the logical form of sentences involving comparative adjectives, which validates many of the more obvious inferences between them. In this connection it is worth noting that other constructions related to comparatives can be dealt with along the same lines. ' $a$ is just as $\mathrm{F}$ as $b$ ', ' $a$ is very $\mathrm{F}$ ', and ' $a$ is too $\mathrm{F}$ to be G' are obviously related constructions, and can be handled in an obviously related way. They are, respectively

$\exists d$ ( $a$ is $\mathrm{F}$ to degree $d$ and $B$ is $\mathrm{F}$ to degree $d$ )

$\exists d(a$ is $\mathrm{F}$ to degree $d$ and $d$ is high in $\mathcal{L}(F))$

\footnotetext{
${ }^{1}$ For precise formulations and technical points see S. Maclane and G. Birkhoff Algebra (New York, Macmillan, 1968), Chapter 14, or, more likely to be on a philosopher's shelf, J. L. Bell and A. B. Slomson Models and Ultraproducts (North Holland, 1969), Chapter 1.
} 
$\exists d(a$ is $\mathrm{F}$ to degree $d$ and Necessarily $(x)(e)(x$ is $\mathrm{G}$ and $x$ is $\mathrm{F}$ to degree $e$ ) $\supset d$ exceeds $e$ in $\mathcal{L}(\mathrm{F})$ )

Inferences between these constructions and comparatives (e.g. ' $a$ is too $\mathrm{F}$ to be $\mathrm{G}, b$ is $\mathrm{G}$, therefore $a$ is more $\mathrm{F}$ than $b$ ') are easily captured by this regimentation. At one time I thought that in order to capture some other facts about comparative degrees ought to conform to something more restricted than partial orderings, in particular that one ought to require degrees to be members of lattices. A lattice is a partial ordering in which for any two elements there is a greatest and a least of all the elements above and below both of them (see the references in footnote 1). But this suggestion seems to me now to be ruled out by the following example.

Imagine that in a society there is both an ecclesiastical and a temporal hierarchy, and that they are independent: there is no convention about how the two kinds of rank are compared and there is no top position common to both. Then 'is of high rank' and 'ranks higher than' both have application, but the partial ordering characterizing them is such that if $a$ is of high ecclesiastical rank and $b$ of high temporal rank then there will be no $c$ such that $c$ outranks both. And we may imagine that the institutions of the society forbid the joint possession of high ecclesiastical and temporal rank, so that it is part of the meaning of 'is of high rank' that this be so. Then $\mathcal{L}$ (is of high rank) is not a lattice. And I do not see how the fact that 'is of high rank' can be analysed as a disjunction can prevent it from being a proper predicate with a proper comparative.

If for any partial ordering $\Omega$ we can form a vague, but intelligible, predicate 'ranks highly in $\Omega$ ', then we can repeat the argument of the last paragraph for this ordering. Then no restrictions could be imposed on the concept of a degree except the fairly mild one I have already postulated, that degrees form partial orderings. But I do not in fact think that this is the case. I think that we should exclude some partial orderings, for example one in which there are just two elements, neither one bearing the ordering relation to the other. Such an ordering could not be construed as that of the degrees of a predicate, because it does not allow for the fact that objects falling under the predicate must have that predicate to a greater degree than objects not falling under it.

Perhaps we can simply require that the partial ordering associated with a predicate allow a division into two classes, all of one bearing the ordering relation to all of the other. This would account for the obvious validity of

(Fx and $\sim$ Fy) $\supset \mathrm{x}$ is F-er than $y$.

I am not convinced, though, either that this is the right way to handle this validity or that it represents the only reasonable further constraint on the partial orderings which represent the degrees of predicates. And I suggest that the interesting question we are left 
with, the successor to Williams' original question, is that of what the right set of constraints (expressed axiomatically or algebraically) on a partial ordering is, in order for it to be interpretable as the set of degrees of an intelligible predicate.

University of Bristol, Queens Road, Bristol BS8 1RJ

(C) ADAM MORTON 1984

\title{
COMMENT
}

\author{
By C. J. F. Williams
}

THE price of Adam Morton's answer to my question may seem excessive, when we consider such simple propositions as 'Sarah has grown' or 'The radiator is heating up'. These propositions are equivalent to 'Sarah is taller than she was' and 'The radiator is hotter than it was'. Morton's analysis of these will then have the form

$\exists d \exists e \exists t$ ( $a$ was $\mathbf{F}$ to degree $d$ at time $t$ and $a$ is $\mathbf{F}$ to degree $e$ now and $t$ is earlier than now and $e$ exceeds $d$ in $\mathcal{L}(\mathrm{F})$ )

I might even want to expand this to

$\exists d \exists e \exists t(a ; x, y)$ ( $x$ was $\mathrm{F}$ to degree $d$ at time $t$ and $y$ is $\mathrm{F}$ to degree $e$ now and $t$ is earlier than now and $e$ exceeds $d$ in $\mathcal{L}(\mathrm{F})$ )

Can growth and warming up be as complicated as this?

One reaction must be dismissed quickly. It will not do to say that growth and every sort of change which involves quantity, whether extensive or intensive, belong to a class of phenomena which cannot be further analysed. We cannot acquiesce in the notion that growth is, as Locke would put it, a simple idea. Whether we say that Sarah has grown or that Sarah overtops Richard we are either way employing the concept taller than; and the connections between the two-place predicable ' - is taller than ... is' and the one place predicable ' - has grown' have to be traced. The cost may be the talk of degrees and partial orderings which Morton suggests, and if this is what it costs, we must pay up. But I am still open to lower bids.

University of Bristol, Queens Road, Bristol BS8 1RJ

() C. J. F. Williams 1984 